\title{
PLANE WAVES IN THERMOELASTICITY WITH ONE RELAXATION TIME
}

\author{
JUN WANG and WEN DONG CHANG
}

(Received 20 November 1999)

\begin{abstract}
We apply the thermoelastic equations with one relaxation time developed by Lord and Shulman (1967) to solve some elastic half-space problems. Laplace transform is used to find the general solution. Problems concerning the ramp-type increase in boundary temperature and stress are studied in detail. Explicit expressions for temperature and stress are obtained for small values of time, where second sound phenomena are of relevance. Numerical values of stress and temperature are calculated and displayed graphically.
\end{abstract}

2000 Mathematics Subject Classification. 35L90, 74A15, 80A17.

1. Introduction. The classical theory of thermoelasticity predicts an infinite speed for heat propagation, which is contrary to the physical observations. To overcome this paradox, many papers have been devoted to the development of the generalized theory of thermoelasticity that predicts a finite speed for heat propagation.

The generalized theory of thermoelasticity developed by Lord and Shulman [3] is based on a modified Fourier's law whose governing system of equations is entirely hyperbolic and hence predicts finite speed for heat propagation. Dhaliwal and Sherief [2] extended this theory to general anisotropic materials. Employing this theory, Sherief [4] obtained the solution for a spherically symmetric problem with a point heat source and Wang and Dhaliwal [6] derived solution for a general threedimensional problem and examined two special cases when an infinite body is acted upon by an impulsive body force and by an impulsive heat source. Detailed references to the developments of generalized thermoelasticity can be found in a nice review paper by Chandrasekharaiah [1].

The aim of this paper is to study the thermoelastic interactions in an elastic halfspace. We employ the thermoelastic equations with one relaxation time developed by Lord and Shulman to solve these problems. Laplace transform is used to find the general solution and then solutions to the problems of ramp-type increase in boundary temperature and in boundary stress are obtained for small values of time. The counterparts of these problems in the classical thermoelasticity have been studied by Sternberg and Chakravorty [5].

2. Formulation of the problem and the general solution. We consider a homogeneous and isotropic elastic solid occupying the half-space $x \geq 0$. The nondimensionalized governing system of equations in thermoelasticity with one relaxation time, in 
the absence of heat sources and body forces, are (see [6])

$$
\begin{gathered}
\beta^{2} \ddot{u}_{i}=\frac{\lambda+\mu}{\mu} u_{j, i j}+u_{i, j j}-b \theta_{, i}, \quad \theta_{, i i}=\dot{\theta}+\tau \ddot{\theta}+\omega\left(\dot{u}_{i, i}+\tau \ddot{u}_{i, i}\right), \\
\sigma_{i j}=\frac{\lambda}{\mu} u_{k, k} \delta_{i j}+u_{i, j}+u_{j, i}-b \theta \delta_{i j},
\end{gathered}
$$

where $\lambda$ and $\mu$ are Lamé constants, $\beta^{2}=(\lambda+2 \mu) / \mu, b=\gamma \theta_{0} / \mu, \omega=\gamma / \rho c, \gamma=$ $(3 \lambda+2 \mu) \alpha_{t}, \alpha_{t}$ is the coefficient of linear thermal expansion, $\rho$ is the mass density, $\theta$ is the temperature deviation above the initial temperature $\theta_{0}, c$ is the specific heat for processes with invariant strain tensor, $u_{i}$ are the components of the displacement vector, $\sigma_{i j}$ are the components of stress tensor, and $\tau$ is the relaxation time.

In the problem under consideration, all quantities depend only on one space coordinate $x$ and time $t$ and hence, we have

$$
\begin{aligned}
u_{1} & =u(x, t), & & u_{2}=0, \quad u_{3}=0, \\
\sigma_{11} & =\sigma(x, t), & & \text { otherwise } \sigma_{i j}=0 .
\end{aligned}
$$

Therefore, the governing equations (2.1) reduce to

$$
\beta^{2} \ddot{u}=\beta^{2} u^{\prime \prime}-b \theta^{\prime}, \quad \theta^{\prime \prime}=\dot{\theta}+\tau \ddot{\theta}+\omega\left(\dot{u}^{\prime}+\tau \ddot{u}^{\prime}\right), \quad \sigma=\beta^{2} u^{\prime}-b \theta,
$$

where the prime denotes the partial derivative with respect to $x$.

In the context of the problem considered, the initial and boundary conditions are

$$
\begin{gathered}
u=\dot{u}=\theta=\dot{\theta}=0, \\
\sigma(0, t)=f(t), \quad \theta(0, t)=g(t), \quad t=0 \\
(\sigma, \theta) \longrightarrow(0,0) \quad \text { as } x \longrightarrow \infty,
\end{gathered}
$$

where $f$ and $g$ are prescribed functions.

Now we apply the Laplace transform, defined by

$$
\bar{f}(x, p)=\int_{0}^{\infty} f(x, t) e^{-p t} d t, \quad \operatorname{Re}(p)>0,
$$

to (2.3) under the homogeneous initial conditions (2.4). Applying the boundary conditions (2.5) and (2.6) and following a standard routine as in [4, 6], we obtain the general solution for the stress and temperature fields in the Laplace domain as

$$
\bar{\theta}(x, p)=\bar{\theta}_{1}(x, p)+\bar{\theta}_{2}(x, p), \quad \bar{\sigma}(x, p)=\bar{\sigma}_{1}(x, p)+\bar{\sigma}_{2}(x, p),
$$

with

$$
\begin{aligned}
& \bar{\theta}_{1}(x, p)=\frac{\bar{g}(p)}{\lambda_{1}^{2}-\lambda_{2}^{2}}\left[\left(\lambda_{1}^{2}-p^{2}\right) e^{-\lambda_{1} x}-\left(\lambda_{2}^{2}-p^{2}\right) e^{-\lambda_{2} x}\right], \\
& \bar{\theta}_{2}(x, p)=-\frac{\left(\lambda_{1}^{2}-p^{2}\right)\left(\lambda_{2}^{2}-p^{2}\right)}{\left(\lambda_{1}^{2}-\lambda_{2}^{2}\right) b p^{2}} \bar{f}(p)\left[e^{-\lambda_{1} x}-e^{-\lambda_{2} x}\right], \\
& \bar{\sigma}_{1}(x, p)=\frac{b p^{2}}{\lambda_{1}^{2}-\lambda_{2}^{2}} \bar{g}(p)\left[e^{-\lambda_{1} x}-e^{-\lambda_{2} x}\right], \\
& \bar{\sigma}_{2}(x, p)=-\frac{\bar{f}(p)}{\lambda_{1}^{2}-\lambda_{2}^{2}}\left[\left(\lambda_{2}^{2}-p^{2}\right) e^{-\lambda_{1} x}-\left(\lambda_{1}^{2}-p^{2}\right) e^{-\lambda_{2} x}\right],
\end{aligned}
$$


where $\lambda_{1}^{2}$ and $\lambda_{2}^{2}$ are the roots of the characteristic equation

$$
\left(\lambda^{2}\right)^{2}-p(1+\epsilon+p+\tau p+\epsilon \tau p) \lambda^{2}+p^{3}(1+\tau p)=0, \quad \epsilon=\frac{\omega b}{\beta^{2}} .
$$

The general solution in $x$ and $t$ coordinates can be found by inverting the Laplace transforms in (2.8) and (2.9), which is a formidable task although it is theoretically possible. Following [4, 6], here we assume that time $t$ is very small, where the second sound phenomenon is of relevance.

For small values of $t$, that is, large values of $p$, we expand $\lambda_{1}$ and $\lambda_{2}$ binomially in ascending powers of $1 / p$ and retain only necessary terms to get

$$
\lambda_{1} \approx a_{11}+a_{10} p, \quad \lambda_{2} \approx a_{21}+a_{20} p,
$$

where

$$
\begin{aligned}
& a_{10}=\frac{1}{2}\left(1+\tau+\epsilon T+c_{o}\right), a_{11}=\frac{(1+\epsilon) c_{0}+(1+\tau+\epsilon \tau)(1+\epsilon)-2}{4 c_{0} a_{10}}, \\
& a_{20}=\frac{1}{2}\left(1+\tau+\epsilon \tau-c_{o}\right), a_{21}=\frac{(1+\epsilon) c_{0}-(1+\tau+\epsilon \tau)(1+\epsilon)-2}{4 c_{0} a_{20}}, \\
& c_{0}=\sqrt{(1+\tau+\epsilon \tau)^{2}-4 \tau}
\end{aligned}
$$

Substituting for $\lambda_{1}$ and $\lambda_{2}$ from (2.11) into (2.9), inverting the Laplace transform, we find that

$$
\begin{aligned}
\theta_{1}(x, t)= & H\left(t-a_{10} x\right) e^{-a_{11} x}\left[c_{10} g\left(t-a_{10} x\right)+\sum_{j=1}^{3} \frac{c_{1 j}}{(j-1) !} \int_{0}^{t-a_{10} x} s^{j-1} g\left(t-a_{10} x-s\right) d s\right] \\
& +H\left(t-a_{20} x\right) e^{-a_{21} x}\left[c_{20} g\left(t-a_{20} x\right)+\sum_{j=1}^{3} \frac{c_{2 j}}{(j-1) !} \int_{0}^{t-a_{20} x} s^{j-1} g\left(t-a_{20} x-s\right) d s\right], \\
\theta_{2}(x, t)= & H\left(t-a_{10} x\right) e^{-a_{11} x}\left[d_{0} f\left(t-a_{10} x\right)+\sum_{i=1}^{5} \frac{d_{i}}{(i-1) !} \int_{0}^{t-a_{10} x} s^{i-1} f\left(t-a_{10} x-s\right) d s\right] \\
& -H\left(t-a_{20} x\right) e^{-a_{21} x}\left[d_{0} f\left(t-a_{20} x\right)+\sum_{i=1}^{5} \frac{d_{i}}{(i-1) !} \int_{0}^{t-a_{20} x} s^{i-1} f\left(t-a_{20} x-s\right) d s\right], \\
\sigma_{1}(x, t)= & b H\left(t-a_{10} x\right) e^{-a_{11} x}\left[b_{0} g\left(t-a_{10} x\right)+b_{1} \int_{0}^{t-a_{10} x} g\left(t-a_{10} x-s\right) d s\right] \\
& -b H\left(t-a_{20} x\right) e^{-a_{21} x}\left[b_{0} g\left(t-a_{20} x\right)+b_{1} \int_{0}^{t-a_{20} x} g\left(t-a_{20} x-s\right) d s\right], \\
& +H\left(t-a_{20} x\right) e^{-a_{21} x}\left[c_{10} f\left(t-a_{20} x\right)+\sum_{j=1}^{3} \frac{c_{1 j}}{(j-1) !} \int_{0}^{t-a_{20} x} s^{j-1} f\left(t-a_{20} x-s\right) d s\right], \\
\sigma_{2}(x, t)= & H\left(t-a_{10} x\right) e^{-a_{11} x}\left[c_{20} f\left(t-a_{10} x\right)+\sum_{j=1}^{3} \frac{c_{2 j}}{(j-1) !} \int_{0}^{t-a_{10} x} s^{j-1} f\left(t-a_{10} x-s\right) d s\right] \\
& \\
&
\end{aligned}
$$


where $H(\cdot)$ is the Heaviside step function,

$$
\begin{aligned}
& b_{0}=c_{0}^{-1}, \quad b_{1}=-\frac{(1+\tau+\epsilon \tau)(1+\epsilon)-2}{c_{0}^{3}}, \\
& c_{i 0}=(-1)^{i-1}\left(a_{i 0}^{2}-1\right) b_{0}, \quad c_{i 1}=(-1)^{i-1}\left(2 a_{i 0} a_{i 1} b_{0}+a_{i 0}^{2} b_{1}-b_{1}\right), \\
& c_{i 2}=(-1)^{i-1}\left(a_{i 1}^{2} b_{0}+2 a_{i 0} a_{i 1} b_{1}\right), \quad c_{i 3}=(-1)^{i-1} a_{i 1}^{2} b_{1}, \quad i=1,2 ; \\
& d_{0}=-\left(a_{10}^{2}-1\right)\left(a_{20}^{2}-1\right) \frac{b_{0}}{b}, \\
& d_{1}=-2\left[\left(a_{10}^{2}-1\right) a_{20} a_{21}+\left(a_{20}^{2}-1\right) a_{10} a_{11}\right] \frac{b_{0}}{b}+\frac{d_{0} b_{1}}{b_{0}}, \\
& d_{2}=-\left(a_{11}^{2} a_{21}^{2}+a_{10}^{2} a_{21}^{2}+4 a_{10} a_{11} a_{20} a_{21}-a_{11}^{2}-a_{21}^{2}\right) \frac{b_{0}}{b}+\left(d_{1}-\frac{d_{0} b_{1}}{b_{0}}\right) \frac{b_{1}}{b_{0}}, \\
& d_{3}=-2 a_{11} a_{21}\left(a_{10} a_{21}+a_{11} a_{20}\right) \frac{b_{0}}{b}+\left(d_{2}-\frac{d_{1} b_{1}}{b_{0}}+\frac{d_{0} b_{1}^{2}}{b_{0}^{2}}\right) \frac{b_{1}}{b_{0}}, \\
& d_{4}=-a_{11}^{2} a_{21}^{2} \frac{b_{0}}{b}-2 a_{11} a_{21}\left(a_{10} a_{21}+a_{11} a_{20}\right) \frac{b_{1}}{b}, \quad d_{5}=-a_{11}^{2} a_{21}^{2} \frac{b_{1}}{b} .
\end{aligned}
$$

Inverting the Laplace transform in (2.8), we find the solutions for the stress and temperature fields as

$$
\theta(x, t)=\theta_{1}(x, t)+\theta_{2}(x, t), \quad \sigma(x, t)=\sigma_{1}(x, t)+\sigma_{2}(x, t) .
$$

3. A ramp-type increase in boundary temperature. In this section, we consider an elastic half-space $x \geq 0$ whose boundary surface $x=0$ is subjected to a ramp-type heating according to the following relation:

$$
\begin{gathered}
f(t)=0, \quad g(t)=\theta_{0} h(t), \\
h(t)=\frac{1}{t_{0}}\left[t-H\left(t-t_{0}\right)\left(t-t_{0}\right)\right]= \begin{cases}0, & t \leq 0, \\
\frac{t}{t_{0},} & 0 \leq t \leq t_{0}, \\
1, & t \geq t_{0},\end{cases}
\end{gathered}
$$

where $\theta_{0}$ is a constant and $t_{0} \geq 0$ is a fixed moment of time.

In this problem, $\theta_{2}(x, t)=\sigma_{2}(x, t)=0$, and we find that

$$
\theta(x, t)=\theta_{1}(x, t), \quad \sigma(x, t)=\sigma_{1}(x, t) .
$$

Using (3.2), we can easily obtain

$$
\int_{0}^{t-a} s^{j-1} h(t-a-s) d s=\frac{1}{t_{0}(j+1) j}\left[(t-a)^{j+1}-H\left(t-t_{0}-a\right)\left(t-t_{0}-a\right)^{j+1}\right] .
$$


Substituting from (3.4) into (2.13), (2.15), and (3.3), we find that

$$
\begin{aligned}
\frac{\theta(x, t)}{\theta_{0}}= & \frac{1}{t_{0}} H\left(t-a_{10} x\right) e^{-a_{11} x} \sum_{j=0}^{3} \frac{c_{1 j}}{(j+1) !}\left[\left(t-a_{10} x\right)^{j+1}-H\left(t-a_{10} x-t_{0}\right)\left(t-a_{10} x-t_{0}\right)^{j+1}\right] \\
+ & \frac{1}{t_{0}} H\left(t-a_{20} x\right) e^{-a_{21} x} \sum_{j=0}^{3} \frac{c_{2 j}}{(j+1) !}\left[\left(t-a_{20} x\right)^{j+1}-H\left(t-a_{20} x-t_{0}\right)\left(t-a_{20} x-t_{0}\right)^{j+1}\right], \\
\frac{\sigma(x, t)}{\theta_{0}}=\frac{b}{t_{0}} H\left(t-a_{10} x\right) e^{-a_{11} x}\left\{\left(t-a_{10} x\right)\left[b_{0}+\frac{1}{2} b_{1}\left(t-a_{10} x\right)\right]\right. & \left.-H\left(t-a_{10} x-t_{0}\right)\left(t-a_{10} x-t_{0}\right)\left[b_{0}+\frac{1}{2} b_{1}\left(t-a_{10} x-t_{0}\right)\right]\right\} \\
-\frac{b}{t_{0}} H\left(t-a_{20} x\right) e^{-a_{21} x}\left\{\left(t-a_{20} x\right)\left[b_{0}+\frac{1}{2} b_{1}\left(t-a_{20} x\right)\right]\right. & \left.-H\left(t-a_{20} x-t_{0}\right)\left(t-a_{20} x-t_{0}\right)\left[b_{0}+\frac{1}{2} b_{1}\left(t-a_{20} x-t_{0}\right)\right]\right\} .
\end{aligned}
$$

4. A ramp-type increase in boundary stress. In this section, we consider an elastic half-space $x \geq 0$ whose boundary surface $x=0$ is subjected to a ramp-type increase in stress according to the following relation:

$$
f(t)=\sigma_{0} h(t), \quad g(t)=0
$$

where $\sigma_{0}$ is a constant and $h(t)$ is defined in (3.2). In this problem, $\theta_{1}(x, t)=\sigma_{1}(x, t)=0$, and we have

$$
\theta(x, t)=\theta_{2}(x, t), \quad \sigma(x, t)=\sigma_{2}(x, t) .
$$

Substituting from (3.4) into (2.14), (2.16), and (4.2), we obtain

$$
\begin{aligned}
\frac{\theta(x, t)}{\sigma_{0}}= & \frac{1}{t_{0}} H\left(t-a_{10} x\right) e^{-a_{11} x} \sum_{i=0}^{5} \frac{d_{i}}{(i+1) !}\left[\left(t-a_{10} x\right)^{i+1}-H\left(t-a_{10} x-t_{0}\right)\left(t-a_{10} x-t_{0}\right)^{i+1}\right] \\
& -\frac{1}{t_{0}} H\left(t-a_{20} x\right) e^{-a_{21} x} \sum_{i=0}^{5} \frac{d_{i}}{(i+1) !}\left[\left(t-a_{20} x\right)^{i+1}-H\left(t-a_{20} x-t_{0}\right)\left(t-a_{20} x-t_{0}\right)^{i+1}\right], \\
\frac{\sigma(x, t)}{\sigma_{0}}= & \frac{1}{t_{0}} H\left(t-a_{10} x\right) e^{-a_{11} x} \sum_{j=0}^{3} \frac{c_{2 j}}{(j+1) !}\left[\left(t-a_{10} x\right)^{j+1}-H\left(t-a_{10} x-t_{0}\right)\left(t-a_{10} x-t_{0}\right)^{j+1}\right] \\
& +\frac{1}{t_{0}} H\left(t-a_{20} x\right) e^{-a_{21} x} \sum_{j=0}^{3} \frac{c_{1 j}}{(j+1) !}\left[\left(t-a_{20} x\right)^{j+1}-H\left(t-a_{20} x-t_{0}\right)\left(t-a_{20} x-t_{0}\right)^{j+1}\right] .
\end{aligned}
$$

5. Numerical results and conclusions. The numerical values of the stress and temperature fields at time $t=0.01$ and $t=0.04$ have been calculated and displayed in 


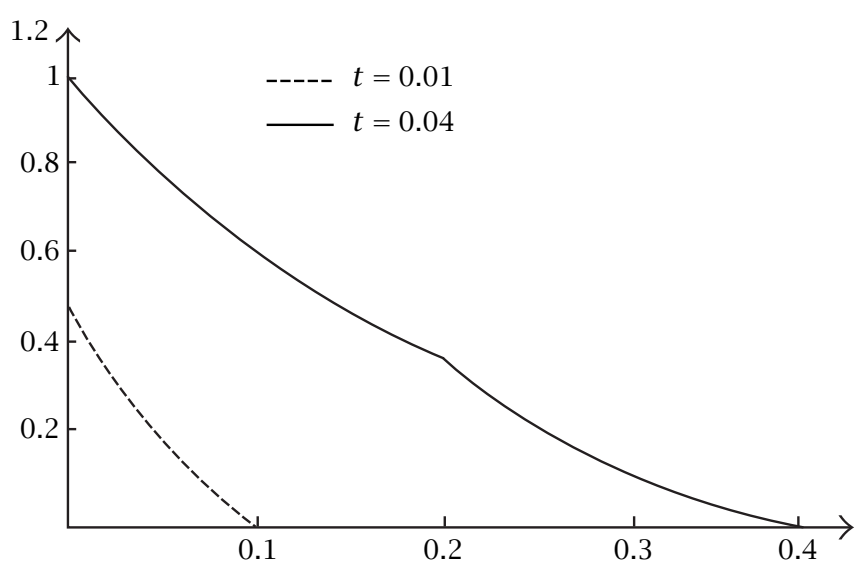

FIGURE 5.1. Temperature $\theta / \theta_{0}$ versus $x$ in the ramp-type heating problem.



FIGURE 5.2. Stress $\sigma / \theta_{0}$ versus $x$ in the ramp-type heating problem.

Figures 5.1, 5.2, 5.3, and 5.4 along the $x$ axis. To obtain these numerical values, we have taken $t_{0}=0.02$, and assumed that $\epsilon=0.0168, b=4.8$, and $\tau=0.1$.

For the case of ramp-type increase in boundary temperature, the numerical values of temperature and stress have been displayed in Figures 5.1 and 5.2, respectively. The magnitude of temperature decreases from its boundary value continuously to zero and vanishes identically after $x \approx 0.1$ for $t=0.01$ and after $x \approx 0.04$ for $t=0.04$. The ramp-type increase in boundary temperature induces a negative stress distribution in the neighborhood of the boundary, but no effect is felt beyond the point $x \approx 0.1$ for $t=0.01$ and beyond the point $x \approx 0.4$ for $t=0.04$.

For the case of ramp-type increase in boundary stress, the numerical values of temperature and stress have been displayed in Figures 5.3 and 5.4, respectively. The result shows that the ramp-type increase in boundary stress induces a very small change of temperature near the boundary. The magnitude of stress decreases quite 


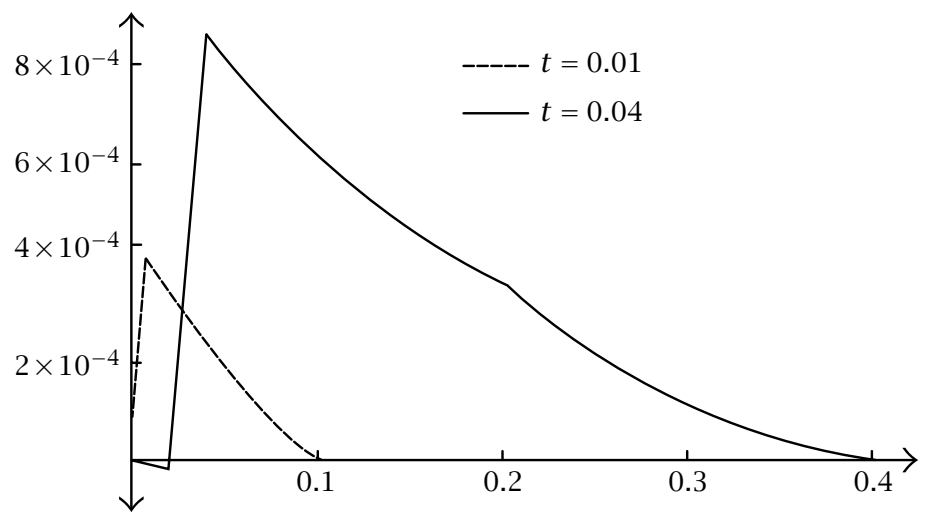

FIGURE 5.3. Temperature $\theta / \sigma_{0}$ versus $x$ in the ramp-type stress problem.



FIGURE 5.4. Stress $\sigma / \sigma_{0}$ versus $x$ in the ramp-type stress problem.

rapidly from its boundary value, but does not vanish up to the point $x \approx 0.1$ for $t=0.01$ and up to the point $x \approx 0.4$ for $t=0.04$. The magnitude of stress in the interval $(0.01,0.1)$ at $t=0.01$ and in the interval $(0.04,0.4)$ at $t=0.04$ is too small to be clearly shown in the graph.

\section{REFERENCES}

[1] D. S. Chandrasekharaiah, Thermoelasticity with second sound: a review, Appl. Mech. Rev. 39 (1986), 355-376. Zbl 588.73006.

[2] R. S. Dhaliwal and H. H. Sherief, Generalized thermoelasticity for anisotropic media, Quart. Appl. Math. 38 (1980), no. 1, 1-8. MR 81j:73082. Zbl 432.73013.

[3] H. W. Lord and Y. Shulman, A generalized dynamical theory of thermoelasticity, J. Mech. Phys. Solids 15 (1967), 299-309. Zbl 156.22702.

[4] H. H. Sherief, Fundamental solution of the generalized thermoelastic problem for small times, J. Thermal Stresses 9 (1986), 151-164.

[5] E. Sternberg and J. G. Chakravorty, On inertia effects in a transient thermoelastic problem, J. Appl. Mech. 26 (1959), 503-509. MR 22\#8877. 
[6] J. Wang and R. S. Dhaliwal, Fundamental solutions of the generalized thermoelastic equations, J. Thermal Stresses 16 (1993), no. 2, 135-161. MR 94a:73011.

Jun Wang: Department of Mathematics and Computer Science, Alabama State UniVERSITY, MONTGOMERY, ALABAMA, USA

E-mail address: junwang@asunet.alasu.edu

Wen Dong Chang: Department of Mathematics and Computer Science, Alabama State UNIVERSITY, MONTGOMERY, ALABAMA, USA

E-mail address: wchang@asunet.a7asu.edu 




Advances in

Operations Research

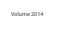

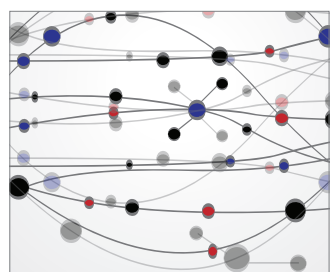

\section{The Scientific} World Journal


International Journal of

Mathematics and

Mathematical

Sciences
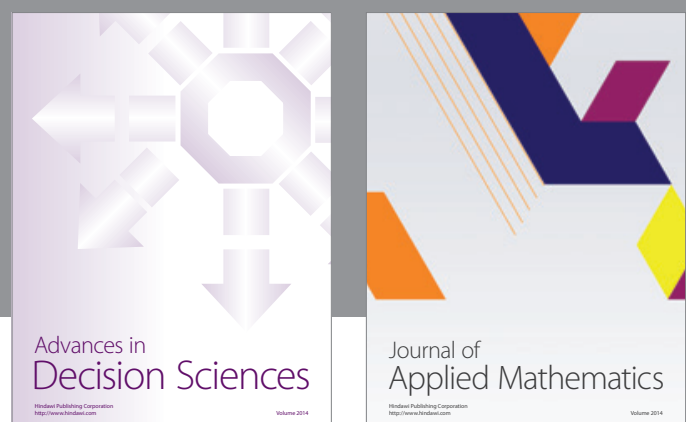

Journal of

Applied Mathematics
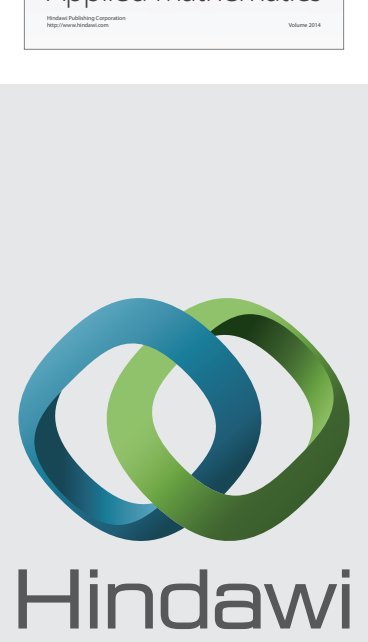

Submit your manuscripts at http://www.hindawi.com


Mathematical Problems in Engineering
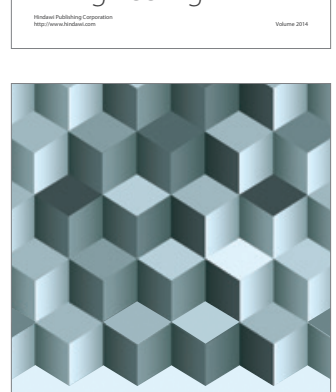

Journal of

Function Spaces
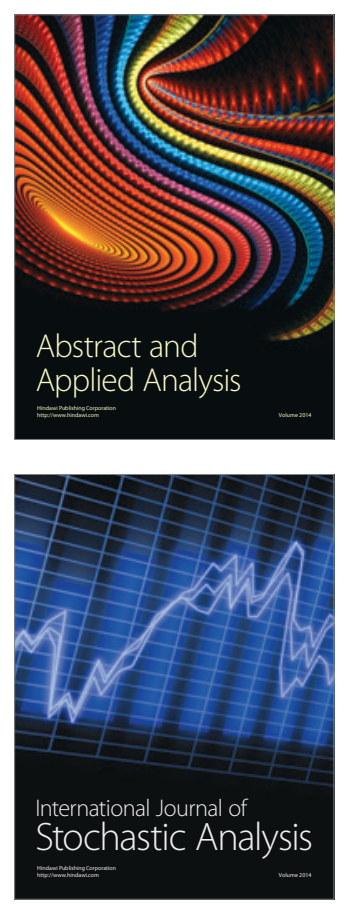

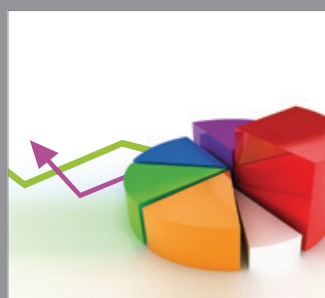

ournal of

Probability and Statistics

Promensencen
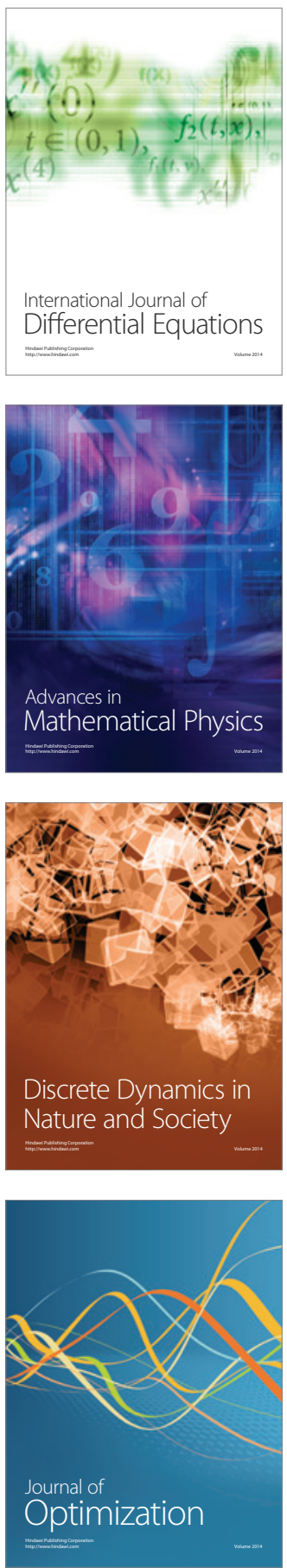